Pacific Journal of Mathematics

RIGHT ORDERABLE GROUPS THAT ARE NOT LOCALLY 


\title{
RIGHT ORDERABLE GROUPS THAT ARE NOT LOCALLY INDICABLE
}

\author{
GeORge M. BergmaN
}

\begin{abstract}
The universal covering group of $\operatorname{SL}(2, \mathbf{R})$ is right orderable, but is not locally indicable; in fact, it contains nontrivial finitely generated perfect subgroups.
\end{abstract}

Introduction. A group $G$ is called right orderable if it admits a total ordering $\leq$ such that $a \leq b \Rightarrow a c \leq b c \quad(a, b, c \in G)$. It is known that a group has such an ordering if and only if it is isomorphic to a group of order-preserving permutations of a totally ordered set [4, Theorem 7.1.2].

A group is called locally indicable if each of its finitely generated nontrivial subgroups admits a nontrivial homomorphism to $\mathbf{Z}$. Every locally indicable group is right orderable (see [4, Theorem 7.3.11]); it was an open question among workers in the area whether the converse was true (equivalent to [9, Problem 1]). This note gives a counterexample, and a modified example showing that a finitely generated right orderable group can in fact be a perfect group.

Related to the characterization of right orderable groups in terms of actions on totally ordered sets is the result that the fundamental group of a manifold $M$ is right orderable if and only if the universal covering space of $M$ can be embedded over $M$ in $M \times \mathbf{R}$. After distributing a preprint of this note, I was informed by W. Thurston and $P$. Kropholler that examples with the same properties were already known among topologists from this point of view (see $\S 6$ below. For another topological use of right ordered groups, in this case locally indicable ones, see [11].) However, as the present examples are easily established and self-contained, they seem worth presenting.

Since the classes of locally indicable groups and of right orderable groups are distinct, it will now be of interest to investigate whether various results that have been proved for the former also hold for the latter; cf. [8], [2, §9], [3, §4].

2. The group $G$. Let $G$ denote the universal covering group of $\operatorname{SL}(2, \mathbf{R})$. Elements of $G$ may be thought of as linear transformations 
of the plane, in which the angle through which each ray is moved is specified, not merely modulo $2 \pi$, but as a real number (in a continuous fashion).

Note that $\operatorname{SL}(2, \mathbf{R})$ acts faithfully on the set of rays through 0 in the plane. Similarly, $G$ acts faithfully on the set of rays through 0 in the infinite-sheeted branched covering $X$ of the plane with branchpoint 0 . This set of rays, "the circle unwound", can be identified with the real line $\mathbf{R}$, and $G$ clearly acts on this set in a way preserving the usual ordering of the line; hence $G$ is right orderable. Members of $G$, thought of as order-preserving maps from $\mathbf{R}$ to itself, have the diagonal periodicity property

$$
f(x+\pi)=f(x)+\pi \quad(x \in \mathbf{R}) .
$$

Note that if $h \in \operatorname{SL}(2, \mathbf{R})$ has a positive eigenvalue $\lambda$, then exactly one of $h$ 's infinitely many liftings to $G$ fixes the rays in $X$ that map into the eigenspace of $\lambda$ (i.e., moves each of these rays through the angle 0 , rather than through $2 \pi, 4 \pi$, etc.). If an element of $\operatorname{SL}(2, \mathbf{R})$ has two positive eigenvalues, then the lifting to $G$ that fixes the rays that map into one eigenspace is also the lifting that fixes the rays mapping into the other. For it is easy to see that an element of $\operatorname{SL}(2, \mathbf{R})$ with at least one positive eigenvalue moves every ray through an angle of magnitude strictly less than $\pi$, hence it cannot be lifted to a map on $X$ that moves one ray by 0 and another by a nonzero multiple of $2 \pi$.

Consider now the following three one-parameter families of elements of $\operatorname{SL}(2, \mathbf{R})$, the first two parametrized by a formal real exponent $r$, the third by a positive real subscript $s$ :

$$
a^{r}=\left(\begin{array}{cc}
1 & -r \\
0 & 1
\end{array}\right), \quad b^{r}=\left(\begin{array}{cc}
1 & 0 \\
r & 1
\end{array}\right), \quad c_{s}=\left(\begin{array}{cc}
s^{1 / 2} & 0 \\
0 & s^{-1 / 2}
\end{array}\right) .
$$

Clearly,

$$
a^{r} a^{r^{\prime}}=a^{r+r^{\prime}}, \quad b^{r} b^{r^{\prime}}=b^{r+r^{\prime}}, \quad c_{s} c_{s^{\prime}}=c_{s s^{\prime}} .
$$

Moreover, we note that

$$
c_{s} a^{r} c_{s}^{-1}=a^{s r}, \quad c_{s}^{-1} b^{r} c_{s}=b^{s r}
$$

and if we define

$$
d=a b a=\left(\begin{array}{cc}
0 & -1 \\
1 & 0
\end{array}\right),
$$


then we have

$$
\begin{gathered}
d^{-1} c_{s} d=c_{s^{-1}}, \\
d^{-1} a^{r} d=b^{r}, \quad d^{-1} b^{r} d=a^{r} .
\end{gathered}
$$

For all real numbers $r$ and positive real numbers $s$, let us define $A^{r}, B^{r}, C_{s}$ to be the unique liftings to $G$ of $a^{r}, b^{r}, d_{s}$ which fix the rays in $X$ lying over the eigenspaces of those elements. We claim that (2) and (3) imply the corresponding equations in $G$ :

$$
\begin{gathered}
A^{r} A^{r^{\prime}}=A^{r+r^{\prime}}, \quad B^{r} B^{r^{\prime}}=B^{r+r^{\prime}}, \quad C_{s} C_{s^{\prime}}=C_{s s^{\prime}}, \\
C_{s} A^{r} C_{s}^{-1}=A^{s r}, \quad C_{s}^{-1} B^{r} C_{s}=B^{s r} .
\end{gathered}
$$

Indeed, by (2) and (3), in each of these five formulas the two sides map to the same element of $\operatorname{SL}(2, \mathbf{R})$, hence it suffices to show that both sides of each equation have fixed rays. The right-hand side of each equation has fixed rays by definition. The left-hand side of the first formula has fixed rays because all real powers of $a$ have a common fixed ray. Hence the same is true of their chosen liftings; corresponding considerations apply to the remaining two formulas of $\left(2^{\prime}\right)$. The left-hand sides of the two equations of $\left(3^{\prime}\right)$ have fixed rays because they are conjugates of elements having fixed rays; this completes the verification of these formulas.

Defining $D$ by the analog of (4):

$$
D=A B A \text {, }
$$

we similarly deduce the analogs of (5) and (6):

$$
\begin{gathered}
D^{-1} C_{s} D=C_{s^{-1}} \\
D^{-1} A^{r} D=B^{r}, \quad D^{-1} B^{r} D=A^{r} .
\end{gathered}
$$

3. The first example. Consider now for any integer $n>1$ the finitely generated subgroup $\left\langle A, B, C_{n}\right\rangle \subseteq G$. Equations $\left(3^{\prime}\right)$ and $\left(5^{\prime}\right)$ show that each of $A, B, C_{n}$ is conjugate in this group to a distinct power of itself, hence any homomorphism $\left\langle A, B, C_{n}\right\rangle \rightarrow \mathbf{Z}$ annihilates these three generators, hence is trivial.

Thus $G$ is not locally indicable.

4. Motivation for the construction. Before finding this example, I wondered whether it might be possible to prove all right orderable groups locally indicable by using the fact that one can write a finitely generated right orderable group $G$ as a group of order-automorphisms $f$ of the real line with $|f(x)-x|$ bounded, and mapping such a group into $\mathbf{R}$ by some measure of the "long-term forward flow" induced 
by each element. Some more subtle measure would then have to be applied to groups whose elements all had zero flow, in this sense. But it turned out that such a flow function, even if nonzero, would not in general be a homomorphism: two order-preserving functions $f$ and $g$ each with "blocked" flow can have composite with nontrivial flow. E.g., this can happen if $f$ is a function which fixes the even integers but pushes all other real numbers a bit forward, and $g$ a function which fixes the odd integers and likewise pushes other values forward.

If such functions have a periodicity property like (1), their behavior can be examined with the help of the induced functions on the circle. Experimentation with convenient cases led to the example described.

5. Further questions, and a modified example. Our above example is equivalent to an affirmative answer to the question, "Is there a nontrivial finitely generated right orderable group $H$ such that the abelianization $H / H^{\prime}$ is a torsion group?" Question 20 of [1] asks whether a nontrivial finitely generated right orderable group can in fact be simple, and Question $20^{\prime}$ asks the intermediate question of whether such an $H$ can be perfect (have trivial abelianization).

The group $\left\langle A, B, C_{n}\right\rangle$ of $\S 3$ is certainly not simple, since it has the central subgroup $\left\langle D^{2}\right\rangle$. It is also quite possibly never perfect: $\left(3^{\prime}\right)$ and $\left(5^{\prime}\right)$ merely tell us that in the abelianization, the images of $A$ and $B$ have exponent $n-1$, and that of $C_{n}$ has exponent 2. (If the integer $n$ is not a square, the possible 2-torsion of the image of $C_{n}$ is indeed realized, for we can map $\left\langle a, b, c_{n}\right\rangle$ to $Z_{2}$ by sending elements with rational entries to 0 , and elements of the form $n^{1 / 2}$ times a rational matrix to 1.) However, we shall now describe a finitely generated perfect subgroup of $G$.

Note that by $\left(4^{\prime}\right)$,

$$
D \in\langle A, B\rangle \text {. }
$$

Conjugating by any element $C_{S}$, and simplifying the left-hand side with the help of $\left(5^{\prime}\right)$ and the right-hand side with the help of $\left(3^{\prime}\right)$, we get

$$
D C_{s^{2}} \in\left\langle A^{s^{-1}}, B^{s}\right\rangle
$$

Taking for $s$ any positive integer $n$, we see that by the two preceding displays, the group $\left\langle A^{n^{-1}}, B\right\rangle$ will contain both $D$ and $D C_{n^{2}}$, hence

$$
C_{n^{2}} \in\left\langle A^{n^{-1}}, B\right\rangle \text {. }
$$

Now consider the group $\left\langle A^{1 / 6}, B\right\rangle$. Since this contains $\left\langle A^{1 / 2}, B\right\rangle$ and $\left\langle A^{1 / 3}, B\right\rangle$, it contains $C_{4}$ and $C_{9}$, hence by $\left(3^{\prime}\right)$, the generators $A^{1 / 6}$ and $B$ are each conjugate in this group both to their 4 th and to 
their 9 th powers. But $4-1=3$ and $9-1=8$ are relatively prime, so each of these generators vanishes in the abelianization, proving this group perfect.

We remark that by $\left(4^{\prime}\right)$ and $\left(6^{\prime}\right)$, the above group can be variously described as

$$
\left\langle A^{1 / 6}, B\right\rangle=\left\langle A^{1 / 6}, B^{1 / 6}\right\rangle=\left\langle A^{1 / 6}, D\right\rangle=\left\langle A^{1 / 2}, B^{1 / 3}\right\rangle .
$$

The same argument shows that $\left\langle A^{1 / m n}, B\right\rangle$ is perfect for any integers $m$ and $n$ such that $m^{2}-1$ and $n^{2}-1$ are relatively prime. But for this relative primality to hold, the product $m n$ must be divisible by 6 , so our example is the simplest of this sort.

In contrast to the above example, it is known that for all real numbers $s \geq 4$, the group $\left\langle a^{s}, b\right\rangle$ is free on the indicated generators [5]. (What is actually shown in [5] is that $\left\langle a^{s}, b^{s}\right\rangle$ is free if $s \geq 2$; but (3) shows that the isomorphism class of $\left\langle a^{s}, b^{t}\right\rangle$ depends only on $s t$. See $[10$, p. 168] for further references.) Hence the same is true of $\left\langle A^{S}, B\right\rangle$.

6. Still more examples and questions. Bill Thurston and Peter Kropholler have pointed out to me an example of a finitely generated perfect right orderable group known to topologists:

$$
\left\langle x, y, z \mid x^{2}=y^{3}=z^{7}=x y z\right\rangle .
$$

That this is perfect can be verified from the presentation. It is right orderable because it has an embedding in the same group $G$ we have been considering, given by

$$
x=A B A=D, \quad y=A^{r} B A^{1-r}, \quad z=y^{-1} x,
$$

where $r$ is a root of $r^{2}-r+2=2 \cos (\pi / 7)$. Here I shall merely sketch a proof that the elements (9) satisfy the relations of (8), and thus generate a nontrivial perfect subgroup of $G$.

In $G, y$ is conjugate to $A B$, which satisfies $(A B)^{3}=(A B A)(B A B)$ $=D D^{D}=D^{2}$ (cf. $\left.\left(6^{\prime}\right)\right)$; so as $D^{2}$ is central in $G$, we also have $y^{3}=D^{2}$. Hence $y^{3}=x^{2}=x y z$. Note that $D^{2}$ represents rotation through an angle of $+\pi$ in the branched covering $X$ of the plane. Now calculation shows that the image of $z$ in $\operatorname{SL}(2, \mathbf{R})$ has eigenvalues $e^{ \pm \pi i / 7}$, hence has 7 th power $-I$, and that it turns rays counterclockwise. Thus after an appropriate change of coordinates in the plane, this element represents a counterclockwise rotation by $\pi / 7$. We claim that, in the same coordinates, the preimage $z=y^{-1} x \in G$ of this element represents a rotation by $\pi / 7$ rather than, say, $15 \pi / 7$. Indeed, this follows from the observation that $y^{-1}$ and $x$ move rays 
in opposite directions, and each through angles $<\pi$ (since they are roots of $D^{ \pm 2}$ ). From this description of $z$ we can see that $z^{7} \in G$ is a rotation through exactly $\pi$ i.e., is $D^{2}$, as desired.

Thurston and Kropholler note that another perfect example is a group of periodic piecewise linear transformations of the line, related to work of R. J. Thompson, and called $\tilde{G}$ in [7]; cf. [6, pp. 54-55].

The question of whether a nontrivial finitely generated right orderable group can be simple remains open. Another open question, [1, Question $21^{\prime}$ ], is whether a nontrivial solvable right orderable group must be locally indicable. I also do not know any examples of nontrivial finitely generated right orderable groups having both finite abelianization and trivial center.

Acknowledgment. This work was done while the author held NSF contract DMS 85-02330.

\section{REFERENCES}

[1] The Black Swamp Problem Book, an ongoing notebook of questions on ordered groups. Copies available from W. Charles Holland (Bowling Green State University), Keeper of the Book.

[2] George M. Bergman, Ordering coproducts of groups and semigroups, J. Algebra, 133 (1990), 313-339.

[3] _ Notes on orderable and right-orderable groups (addenda to "Ordering coproducts of groups and semigroups"), unpublished note, available from the author.

[4] Roberta Botto Mura and Akbar H. Rhemtulla, Orderable Groups, Dekker Lecture Notes in Pure and Appl. Math., v. 27, 1977. MR 58 \#10652.

[5] Joël Lee Brenner, Quelques groupes libres de matrices, C. R. Acad. Sci. Paris, 241 (1955), 1689-1691. MR 17, p. 824.

[6] Kenneth S. Brown, Finiteness properties of groups, J. Pure Appl. Algebra, 44 (1987), 45-75.

[7] Étienne Ghys et Vlad Sergiescu, Sur un groupe remarquable de difféomorphismes du cercle, Commentarii Math. Helvet., 62 (1987) 185-239.

[8] James Howie, On locally indicable groups, Math. Zeitschr., 180 (1982), 445451.

[9] Ali I. Kokorin and Valerij M. Kopytov, Linearly Ordered Groups (Russian), Nauka, Moscow, 1972. Translated as Fully Ordered Groups, Halsted Press, NY, 1974.

[10] Roger Lyndon and Paul Schupp, Combinatorial Group Theory, Ergebnisse der Mathematik, vol. 89, Springer-Verlag, 1977.

[11] Lee Paul Neuwirth, Trivial knots with arbitrary projection, J. Australian Math. Soc., 7 (1967), 481-489.

Received October 10, 1989. 


\title{
PACIFIC JOURNAL OF MATHEMATICS EDITORS
}

\author{
V. S. VARADARAJAN \\ (Managing Editor) \\ University of California \\ Los Angeles, CA 90024-1555-05 \\ Herbert Clemens \\ University of Utah \\ Salt Lake City, UT 84112 \\ THOMAS ENRIGHT \\ University of California, San Diego \\ La Jolla, CA 92093
}

R. FINN

Stanford University

Stanford, CA 94305

Hermann FlaschKa

University of Arizona

Tucson, AZ 85721

VAUGHaN F. R. JoNes

University of California

Berkeley, CA 94720

Steven Kerckhoff

Stanford University

Stanford, CA 94305

\section{C. MOORE}

University of California

Berkeley, CA 94720

Martin ScharlemanN

University of California

Santa Barbara, CA 93106

HAROLD STARK

University of California, San Diego

La Jolla, CA 92093
R. ARENS
E. F. BECKENBACH
F. WolF
K. YoshidA

\section{ASSOCIATE EDITORS}

\begin{tabular}{ll}
\multicolumn{1}{c}{ SUD-1982) } & \multicolumn{1}{c}{$(1904-1989)$} \\
UNIVERSITY OF ARIZONA & UNIVERSITY OF OREGON \\
UNIVERSITY OF BRITISH COLUMBIA & UNIVERSITY OF SOUTHERN CALIFORNIA \\
CALIFORNIA INSTITUTE OF TECHNOLOGY & STANFORD UNIVERSITY \\
UNIVERSITY OF CALIFORNIA & UNIVERSITY OF HAWAII \\
MONTANA STATE UNIVERSITY & UNIVERSITY OF TOKYO \\
UNIVERSITY OF NEVADA, RENO & UNIVERSITY OF UTAH \\
NEW MEXICO STATE UNIVERSITY & WASHINGTON STATE UNIVERSITY \\
OREGON STATE UNIVERSITY & UNIVERSITY OF WASHINGTON \\
\hline
\end{tabular}

The Supporting Institutions listed above contribute to the cost of publication of this Journal, but they are not owners or publishers and have no responsibility for its content or policies.

\begin{abstract}
Mathematical papers intended for publication in the Pacific Journal of Mathematics should be in typed form or offset-reproduced (not dittoed), double spaced with large margins. Please do not use built up fractions in the text of the manuscript. However, you may use them in the displayed equations. Underline Greek letters in red, German in green, and script in blue. The first paragraph must be capable of being used separately as a synopsis of the entire paper. In particular it should contain no bibliographic references. Please propose a heading for the odd numbered pages of less than 35 characters. Manuscripts, in triplicate, may be sent to any one of the editors. Please classify according to the 1980 Mathematics Subject Classification (1985 Revision) scheme which can be found in the December index volumes of Mathematical Reviews. Supply name and address of author to whom proofs should be sent. All other communications should be addressed to the managing editor, or Elaine Barth, University of California, Los Angeles, California 90024-1555-05.

There are page-charges associated with articles appearing in the Pacific Journal of Mathematics. These charges are expected to be paid by the author's University, Government Agency or Company. If the author or authors do not have access to such Institutional support these charges are waived. Single authors will receive 50 free reprints; joint authors will receive a total of 100 free reprints. Additional copies may be obtained at cost in multiples of 50 .
\end{abstract}

The Pacific Journal of Mathematics (ISSN 0030-8730) is published monthly except for July and August. Regular subscription rate: $\$ 190.00$ a year (10 issues). Special rate: $\$ 95.00$ a year to individual members of supporting institutions.

Subscriptions, orders for numbers issued in the last three calendar years, and changes of address should be sent to Pacific Journal of Mathematics, P.O. Box 969, Carmel Valley, CA 93924, U.S.A. Old back numbers obtainable from Kraus Periodicals Co., Route 100, Millwood, NY 10546.

The Pacific Journal of Mathematics at P.O. Box 969, Carmel Valley, CA 93924 (ISSN 0030-8730) is published monthly except for July and August. Second-class postage paid at Carmel Valley, California 93924, and additional mailing offices. Postmaster: send address changes to Pacific Journal of Mathematics, P.O. Box 969, Carmel Valley, CA 93924.

PUBLISHED BY PACIFIC JOURNAL OF MATHEMATICS, A NON-PROFIT CORPORATION Copyright (C) 1991 by Pacific Journal of Mathematics 